\title{
A TIME-DEPENDENT BEST CHOICE PROBLEM WITH COSTS AND RANDOM LIFETIME IN ORGAN TRANSPLANTS
}

Abstract. This paper develops and analyzes a time-dependent optimal stopping problem and its application to the decision making process concerning organ transplants. Offers (organs for transplant) appear at jump times of a Poisson process. The values of the offers are i.i.d. random variables with a known distribution function. These values express the degree of histocompatibility between the donor and the recipient. The sequence of offers is independent of the jump times of the Poisson process. The decision about acceptance or rejection must be made at the time of appearance of the offer. When the offer is accepted, the decision process terminates and a reward is gained. The reward depends on the value of the selected offer, a discount function and a cost function related to aversion to risk. Otherwise, the decision process is continued until a random time $T$. After this time the reward is equal to zero. The aim of the decision maker is to maximize the expected mean reward. A first-order differential equation for the optimal mean reward is obtained.

1. Introduction. Organizations such as United Network for Organ Sharing (UNOS) operating in USA are responsible for managing the national organ denotation and allocation system. However, the final decision about acceptance of a particular organ (in this case a kidney) is usually left to the transplant surgeon and/or physician responsible for the care of the candidates (see UNOS [27]). This decision is based on the personal experience of the physician and depends on medical judgment regarding the suitability of the organ being offered for specific candidates. In the litera-

2010 Mathematics Subject Classification: Primary 60G40; Secondary $92 B 99$.

Key words and phrases: Elfving problem, Poisson process, random horizon, function of costs, organ transplants, aversion to risk. 
ture, some models are presented helping physicians to make these decisions (for example see Alagoz et al. [1] or David and Yechiali [11]). However, these works do not take into consideration the patient's aversion to risk. In this work we propose a model that includes this factor and as such is more related to the patient's individual preferences.

More precisely, we consider the following problem. The decision about acceptance or rejection of a kidney is mainly based on the degree of histocompatibility between the donor and the recipient and the time that the potential recipient has been under medical care (for example, this can mean the duration of dialysis treatment). One of the relevant criterions of the compatibility is the match-level in the HL-A antigen system. Five matchlevels are defined. One can compute the probability of any match-level between a given recipient and a random graft. With each match-level we associate a value, for example, the probability of graft survival for at least one year or expected lifetime of the graft (see David and Yechiali [11] for more details).

Moreover, new research (Chong et al. [6]) shows that patients with endstage liver disease are less risk averse than those with earlier stages of disease. This means that patients with end-stage liver disease (and hence their physicians, deciding about acceptance or rejection of the organ) are more inclined to take a risk and accept an organ with a lower quality or a lower match-level even if the probability of an immediate death is relatively large, compared to patients who are in better condition.

On the other hand, there are studies confronting experimental results with the theoretical solution of the classical secretary problem showing that real-life decision makers have a tendency to shorten the time of observation, and consequently accept an offer that is worse on average than the one given by the optimal strategy obtained from the mathematical model (see Szajowski [26] for an extensive bibliography). The reason of this divergence can be a wrong fit of the model to the data or ignoring some additional factors like a feeling of risk (this factor was introduced in Szajowski [26]).

Hence, it seems reasonable to extend the model and introduce an unobservable random variable related to reduction of risk aversion and a function corresponding to acceptance of the organ with a lower match-level.

In this paper we provide physicians with a mathematical tool specifying (for each match-level in the HL-A antigen system, the recipient's time on dialysis, the level of aversion to risk and random time of death) whether to accept the organ for transplantation or to reject it and wait for a possibly better offer in the future.

From the mathematical point of view our model is a generalization of the problems considered by Elfving [14, David and Yechiali [11] and Fer- 
enstein and Krasnosielska [16] to the case of an optimal stopping problem with costs, a function related to aversion to risk and a random horizon. Elfving [14] analyzed an optimal stopping problem with a discount function and Poisson-type arrival of offers. He obtained a differential equation which allows one to compute the optimal mean reward. David and Yechiali [11] considered a generalization of the Elfving problem to the case of an optimal stopping problem with a renewal process and an underlying lifetime distribution. They gave an explicit solution for the case with a homogeneous Poisson process, constant discount function and Gamma distribution lifetime. Next, they used this solution to determine the optimal policy. They presented detailed calculations based on actual kidney transplant data. Ferenstein and Krasnosielska [16] generalized the theorem concerning the optimal stopping problem with a random horizon considered in Samuel-Cahn [24] to the Poisson case and applied it to the Elfving problem with an additional random horizon.

In comparison with the model of David and Yechiali [11] and Ferenstein and Krasnosielska [16], we additionally introduce an unobservable random variable and a function related to the changes in patients' risk aversion level. However, the construction of the solution of the model uses similar methods to those in Ferenstein and Krasnosielska [16].

Since our model also has other, non-medical applications, we will consider a more general problem than the one presented above. The other applications and related papers are discussed in the concluding section.

2. The model. The basic assumptions and notations follow those of Chow et al. [7] and Ferenstein and Krasnosielska [16]. Let $(\Omega, \mathcal{F}, \mathcal{P})$ be a probability space on which all random variables are considered. Let $Y_{1}, Y_{2}$, ... be independent, non-negative random variables with distribution function $F$ continuous from the left and with $E\left(Y_{1}\right)=\mu<\infty$, observed at the jump times $\tau_{1}<\tau_{2}<\cdots$ of a homogeneous Poisson process with intensity 1 ; set $Y_{0}=d, 0 \leq d<\infty, \tau_{0}=0$. Furthermore, let $T$ be an extended positive random variable with a known distribution function $F_{T}$ continuous from the right and let $\zeta$ be a positive random variable with a known distribution function $F_{\zeta}$ continuous from the right and with $E(\zeta)<\infty$, where $T$ and $\zeta$ are independent. We assume that the sequences of $Y$ 's and $\tau$ 's are independent, and they are independent of $T$ and $\zeta$. Let $\mathcal{F}_{n}=\sigma\left(Y_{1}, \ldots, Y_{n}, \tau_{1}, \ldots, \tau_{n}\right)$, $\mathcal{F}_{n}^{*}=\sigma\left(\mathcal{F}_{n}, \mathbb{I}\left(T>\tau_{1}\right), \ldots, \mathbb{I}\left(T>\tau_{n}\right)\right), n=1,2, \ldots, \mathcal{F}_{0}=\mathcal{F}_{0}^{*}=\{\emptyset, \Omega\}$, $\mathcal{F}_{\infty}=\sigma\left(\bigcup_{n=0}^{\infty} \mathcal{F}_{n}\right), \mathcal{F}_{\infty}^{*}=\sigma\left(\bigcup_{n=0}^{\infty} \mathcal{F}_{n}^{*}\right)$, where $\mathbb{I}(A)$ is the indicator function of the event $A$. Let $\mathcal{T}$ and $\mathcal{T}^{*}$ be the sets of Markov times adapted to $\left\{\mathcal{F}_{n}\right\}_{n=0}^{\infty}$ and $\left\{\mathcal{F}_{n}^{*}\right\}_{n=0}^{\infty}$, respectively. Our aim is to find $\sup _{t^{*} \in \mathcal{T}^{*}} E \tilde{X}_{t^{*}}^{*}$, where

$$
\tilde{X}_{n}^{*}=\left(Y_{n} r\left(\tau_{n}\right)+c_{1}\left(\tau_{n}\right)-\mathbb{I}\left(\zeta \leq \tau_{n}\right) c_{2}\left(\tau_{n}\right)\right) \mathbb{I}\left(T>\tau_{n}\right)
$$


for $n=0,1, \ldots, \tilde{X}_{\infty}^{*}=\lim \sup _{n \rightarrow \infty} \tilde{X}_{n}^{*}$ and $r(\cdot), c_{1}(\cdot)$ and $c_{2}(\cdot)$ are Borel functions. We assume that $r(\cdot)$ is non-increasing and continuous from the right with $r(0)=1, r(s)>0$ for $s \in[0, U)$ and $r(s)=0$ elsewhere. We also assume that $U<\infty$ and $\left|c_{1}(s)\right| \leq b$ and $\left|c_{2}(s)\right| \leq b$ for $s \geq 0$, where $b$ is some constant. Moreover, we assume that the $\operatorname{limits}_{\lim _{x \rightarrow \infty}} c_{1}(x)$ and $\lim _{x \rightarrow \infty} c_{2}(x)$ exist and are finite. Denote

$$
\tilde{\varphi}(s)=\left(c_{1}(s)-c_{2}(s) F_{\zeta}(s)\right) \bar{F}_{T}(s), \quad \text { where } \quad \bar{F}_{T}(s)=1-F_{T}(s) .
$$

Assume that $\tilde{\varphi}(\cdot)$ is non-increasing or increasing on $(\tilde{U}, \infty)$, where $\tilde{U}=$ $\min \left\{U, U_{1}\right\}$ and $U_{1}=\inf \left\{s>0: \bar{F}_{T}(s)=0\right\}$. Moreover, assume that the function $\tilde{\varphi}(\cdot)$ is either

- continuous on $[0, \infty)$, or

- right-continuous on $[0, \infty)$, increasing or non-increasing on $(0, \infty)$, and $\int_{0}^{\infty}|\tilde{\varphi}(s)| d s<\infty$.

Let the set of all points of non-differentiability of $F(s)$ and discontinuity of the functions $r(s) \bar{F}_{T}(s)$ and $\tilde{\varphi}(s), s \in \mathbb{R}$, be finite. Moreover, if $F$ is discontinuous, then we assume that there exist $l<\infty$ and $0=v_{0}<v_{1}<$ $\cdots<v_{l}=U$ such that in each interval $\left(v_{i}, v_{i+1}\right), i=0,1, \ldots, l-1$, the function

$$
z \mapsto \frac{\sup _{t \in \mathcal{T}} E\left(Y_{t} r\left(z+\tau_{t}\right) \bar{F}_{T}\left(z+\tau_{t}\right)+\tilde{\varphi}\left(z+\tau_{t}\right)\right)-\tilde{\varphi}(z)}{\bar{F}_{T}(z) r(z)}
$$

has exactly one of the following properties: it is increasing, constant or decreasing. This property will be useful in derivation of equation (2.8).

REMARK 2.1. Note that $Y$ 's can be interpreted as probabilities of graft survival for at least one year, $r(\cdot)$ as a discount function related to duration of dialysis treatment, $\xi$ as a random time of reduction of aversion to risk, $c_{2}(\cdot)$ as a function related to the level of aversion to risk, $T$ as a time after which the recipient cannot obtain any organ for transplant because of poor condition (death in particular). Moreover, note that if $c_{1}(s)=0$ for all $s \geq 0$ and $c_{2}(\cdot)$ is a constant function, $d=0$, then the model formulated in this section is consistent with the problem presented in the introduction.

REMARK. If the functions $c_{1}(\cdot)$ and $c_{2}(\cdot)$ are as in Remark 2.1, $\xi$ has a uniform distribution on $[0, U]$, and $T$ has an exponential distribution with parameter, then the assumptions of the model are satisfied.

Define

$$
X_{n}^{*}=\left(Y_{n} r\left(\tau_{n}\right)+\varphi\left(\tau_{n}\right)\right) \mathbb{I}\left(T>\tau_{n}\right), \quad n=0,1, \ldots,
$$

where $\varphi\left(\tau_{n}\right)=c_{1}\left(\tau_{n}\right)-c_{2}\left(\tau_{n}\right) F_{\zeta}\left(\tau_{n}\right)$ and $X_{\infty}^{*}=\limsup _{n \rightarrow \infty} X_{n}^{*}$. Now, we show that $\tilde{X}_{\infty}^{*}=X_{\infty}^{*}$. Since $E\left(\sum_{n=0}^{\infty} Y_{n} r\left(\tau_{n}\right)\right)<\infty$, we have

$$
\lim _{n \rightarrow \infty} Y_{n} r\left(\tau_{n}\right)=0 .
$$


Note that the limits $\lim _{n \rightarrow \infty} \mathbb{I}\left(T>\tau_{n}\right)$ and $\lim _{n \rightarrow \infty} \mathbb{I}\left(\zeta \leq \tau_{n}\right)$ exist and are finite, because $\mathbb{I}\left(T>\tau_{n}\right) \geq \mathbb{I}\left(T>\tau_{n+1}\right)$ and $\mathbb{I}\left(\zeta \leq \tau_{n}\right) \leq \mathbb{I}\left(\zeta \leq \tau_{n+1}\right)$ for each $n \geq 0$. Moreover, $\lim _{n \rightarrow \infty} \mathbb{I}\left(\zeta \leq \tau_{n}\right)=1$ and $\lim _{n \rightarrow \infty} F_{\zeta}\left(\tau_{n}\right)=1$. Hence, from the assumptions of the model we have $\tilde{X}_{\infty}^{*}=\lim _{n \rightarrow \infty} \tilde{X}_{n}^{*}=$ $\lim _{n \rightarrow \infty} X_{n}^{*}=X_{\infty}^{*}$. Since $\left\{\tilde{X}_{n}^{*}\right\}_{n=0}^{\infty}$ is not adapted to the filtration $\left\{\mathcal{F}_{n}^{*}\right\}_{n=0}^{\infty}$, the reward $\tilde{X}_{n}^{*}$ should be replaced by $X_{n}^{*}$, because for each $t^{*} \in \mathcal{T}^{*}$ we have

$$
\begin{aligned}
E\left(\tilde{X}_{t^{*}}^{*}\right)=E\left(\sum_{n=0}^{\infty} \mathbb{I}\left(t^{*}=n\right) \tilde{X}_{n}^{*}\right)+E\left(\mathbb{I}\left(t^{*}=\infty\right) \tilde{X}_{\infty}^{*}\right) \\
=\sum_{n=0}^{\infty} E\left(\mathbb{I}\left(t^{*}=n\right) E\left(\tilde{X}_{n}^{*} \mid \mathcal{F}_{n}^{*}\right)\right)+E\left(\mathbb{I}\left(t^{*}=\infty\right) X_{\infty}^{*}\right) \\
=\sum_{n=0}^{\infty} E\left(\mathbb{I}\left(t^{*}=n\right) \mathbb{I}\left(T>\tau_{n}\right)\left(Y_{n} r\left(\tau_{n}\right)+c_{1}\left(\tau_{n}\right)-c_{2}\left(\tau_{n}\right) E\left(\mathbb{I}\left(\zeta \leq \tau_{n}\right) \mid \mathcal{F}_{n}^{*}\right)\right)\right) \\
\quad+E\left(\mathbb{I}\left(t^{*}=\infty\right) X_{\infty}^{*}\right) \\
=\sum_{n=0}^{\infty} E\left(\mathbb{I}\left(t^{*}=n\right)\left(Y_{n} r\left(\tau_{n}\right)+\varphi\left(\tau_{n}\right)\right) \mathbb{I}\left(T>\tau_{n}\right)\right)+E\left(\mathbb{I}\left(t^{*}=\infty\right) X_{\infty}^{*}\right)=E\left(X_{t^{*}}^{*}\right) .
\end{aligned}
$$

The theorem below is a modification of Theorem 2.1 of Ferenstein and Krasnosielska [16] to a problem with costs and with a random horizon which is an extended real random variable. The idea of transforming an optimal stopping problem with a random horizon to an optimal stopping problem with a modified structure of rewards is based on the paper of SamuelCahn [24].

TheOREM 2.2. Let $Y_{1}, Y_{2}, \ldots$ be i.i.d. non-negative random variables independent of the jump times $\tau_{1}, \tau_{2}, \ldots$ of a Poisson process. Let $Y_{0}=d$, $0 \leq d<\infty, \tau_{0}=0$. Let $T$ be an extended positive random variable independent of the sequences of $Y$ 's and $\tau$ 's, with the distribution function $F_{T}$, and $\bar{F}_{T}(\cdot)=1-F_{T}(\cdot)$. Let $r:[0, \infty) \rightarrow \mathbb{R}$ and $\varphi:[0, \infty) \rightarrow \mathbb{R}$ be Borel functions, $X_{n}^{*}=\left(Y_{n} r\left(\tau_{n}\right)+\varphi\left(\tau_{n}\right)\right) \mathbb{I}\left(T>\tau_{n}\right), n=0,1, \ldots$, and $X_{\infty}^{*}=\lim _{\sup _{n \rightarrow \infty}} X_{n}^{*}$. Assume that the limit $\lim _{n \rightarrow \infty}\left(Y_{n} r\left(\tau_{n}\right)+\varphi\left(\tau_{n}\right)\right)$ exists and is finite, and $E\left(\sup _{n \in \mathbb{N} \cup \infty}\left(X_{n}^{*}\right)^{+}\right)<\infty$. Then

$$
\sup _{t^{*} \in \mathcal{T}^{*}} E\left(X_{t^{*}}^{*}\right)=\sup _{t \in \mathcal{T}} E\left(X_{t}\right),
$$

where $X_{n}=\left(Y_{n} r\left(\tau_{n}\right)+\varphi\left(\tau_{n}\right)\right) \bar{F}_{T}\left(\tau_{n}\right), n=0,1, \ldots$, and $X_{\infty}=\lim \sup _{n \rightarrow \infty} X_{n}$.

Proof. Since $\left\{\mathbb{I}\left(T>\tau_{n}\right)\right\}_{n=0}^{\infty}$ is not measurable with respect to $\left\{\mathcal{F}_{n}\right\}_{n=0}^{\infty}$, using the properties of conditional expectation, we have, for each $t \in \mathcal{T}$, 


$$
\begin{aligned}
E\left(X_{t}^{*}\right) & =E\left(\sum_{n=0}^{\infty} X_{n}^{*} \mathbb{I}(t=n)\right)+E\left(\mathbb{I}(t=\infty) X_{\infty}^{*}\right) \\
& =E\left(\sum_{n=0}^{\infty} X_{n} \mathbb{I}(t=n)\right)+E\left(\mathbb{I}(t=\infty) X_{\infty}^{*}\right) .
\end{aligned}
$$

Now, we need to show that $E\left(\mathbb{I}(t=\infty) X_{\infty}^{*}\right)=E\left(\mathbb{I}(t=\infty) X_{\infty}\right)$. From the assumptions of the theorem we have, for $t \in \mathcal{T}$,

$$
\begin{aligned}
E\left(\mathbb{I}(t=\infty) X_{\infty}^{*}\right) & =E\left(\mathbb{I}(t=\infty) \limsup _{n \rightarrow \infty} X_{n}^{*}\right) \\
& =E\left(\mathbb{I}(t=\infty) \lim _{n \rightarrow \infty}\left(Y_{n} r\left(\tau_{n}\right)+\varphi\left(\tau_{n}\right)\right) \lim _{n \rightarrow \infty}\left(\mathbb{I}\left(T>\tau_{n}\right)\right)\right) \\
& =E\left(\mathbb{I}(t=\infty) \lim _{n \rightarrow \infty}\left(Y_{n} r\left(\tau_{n}\right)+\varphi\left(\tau_{n}\right)\right)\right) P(T=\infty) .
\end{aligned}
$$

On the other hand,

$$
\begin{aligned}
E\left(\mathbb{I}(t=\infty) X_{\infty}\right) & =E\left(\mathbb{I}(t=\infty) \limsup _{n \rightarrow \infty}\left(\left(Y_{n} r\left(\tau_{n}\right)+\varphi\left(\tau_{n}\right)\right) \bar{F}_{T}\left(\tau_{n}\right)\right)\right) \\
& =E\left(\mathbb{I}(t=\infty) \lim _{n \rightarrow \infty}\left(Y_{n} r\left(\tau_{n}\right)+\varphi\left(\tau_{n}\right)\right) \lim _{n \rightarrow \infty} \bar{F}_{T}\left(\tau_{n}\right)\right) \\
& =E\left(\mathbb{I}(t=\infty) \lim _{n \rightarrow \infty}\left(Y_{n} r\left(\tau_{n}\right)+\varphi\left(\tau_{n}\right)\right)\right) P(T=\infty) .
\end{aligned}
$$

Hence, $E\left(\mathbb{I}(t=\infty) X_{\infty}^{*}\right)=E\left(\mathbb{I}(t=\infty) X_{\infty}\right)$. Therefore, from 2.2) we have $E\left(X_{t}^{*}\right)=E\left(X_{t}\right)$ and consequently $\sup _{t \in \mathcal{T}} E\left(X_{t}^{*}\right)=\sup _{t \in \mathcal{T}} E\left(X_{t}\right)$. Moreover, since $\mathcal{T} \subset \mathcal{T}^{*}$, we have

$$
\sup _{t \in \mathcal{T}} E\left(X_{t}^{*}\right) \leq \sup _{t^{*} \in \mathcal{T}^{*}} E\left(X_{t^{*}}^{*}\right)
$$

Now, we will prove the reverse inequality. Set $\mathbb{I}\left(T>\tau_{n}\right)=\xi_{n}$ and $Z_{n}^{*}=$ $\left(Y_{n}, \tau_{n}, \xi_{n}\right), n=0,1, \ldots$ Note that $\left\{Z_{n}^{*}, \mathcal{F}_{n}^{*}\right\}_{n=0}^{\infty}$ is a homogeneous Markov chain with the state space $(E, \mathcal{E})$, where $E=[0, \infty) \times[0, \infty) \times\{0,1\}$. Moreover, using the fact that $Y_{i}$ are i.i.d. random variables, we deduce that $E\left(\operatorname{esssup}_{t^{*} \in \mathcal{T}_{n+1}^{*}} E\left(X_{t^{*}}^{*} \mid \mathcal{F}_{n+1}^{*}\right) \mid \mathcal{F}_{n}^{*}\right)$ is a function of $\tau_{n}$ and $\xi_{n}$ only, say $V^{*}\left(\tau_{n}, \xi_{n}\right)$. Therefore, the Markov time

$$
\sigma^{*}=\inf \left\{n \geq 0: X_{n}^{*} \geq V^{*}\left(\tau_{n}, \xi_{n}\right)\right\}
$$

is optimal in $\mathcal{T}^{*}$, where by definition $\inf \emptyset=+\infty$, that is, $\sup _{t^{*} \in \mathcal{T}^{*}} E\left(X_{t^{*}}^{*}\right)=$ $E\left(X_{\sigma^{*}}^{*}\right)$. Define the function $h(y, s, w)=(y r(s)+\varphi(s)) w$ for $(y, s, w) \in E$. Let

$$
B=\left\{(y, s, w) \in E: h(y, s, w) \geq V^{*}(s, w)\right\}
$$

Hence,

$$
\sigma^{*}=\inf \left\{n \geq 0:\left(Y_{n}, \tau_{n}, \xi_{n}\right) \in B\right\} .
$$

Define $S=\{(y, s, 1) \in E\}, \bar{B}=E \backslash B, C=\{(y, s):(y, s, 1) \in B\}$ and $\bar{C}=\{(y, s):(y, s, 1) \in \bar{B}\}$. Note that $B \cap S=C \times\{1\}$ and $\bar{B} \cap S=$ $\bar{C} \times\{1\}$. Moreover, for each $k \geq 0$ we have $\mathbb{I}\left(\xi_{k}=1\right)=\mathbb{I}\left(\left(Y_{k}, \tau_{k}, \xi_{k}\right) \in S\right)=$ 
$\mathbb{I}\left(\left(Y_{k}, \tau_{k}, \xi_{k}\right) \in S\right) \cdot \ldots \cdot \mathbb{I}\left(\left(Y_{0}, \tau_{0}, \xi_{0}\right) \in S\right)$ and $X_{k}^{*}=X_{k}^{*} \mathbb{I}\left(\xi_{k}=1\right)$. Note that

$$
X_{\sigma^{*}}^{*}=\sum_{n=0}^{\infty} X_{n}^{*} \mathbb{I}\left(\sigma^{*}=n\right)+\limsup _{n \rightarrow \infty} X_{n}^{*} \mathbb{I}\left(\sigma^{*}=\infty\right)
$$

and

$$
\begin{aligned}
\sum_{n=0}^{\infty} X_{n}^{*} \mathbb{I}\left(\sigma^{*}=n\right)=\sum_{n=0}^{\infty} X_{n}^{*} \mathbb{I}\left(\left(Y_{n}, \tau_{n}, \xi_{n}\right) \in S\right) \mathbb{I}\left(\left(Y_{0}, \tau_{0}, \xi_{0}\right) \notin B\right) \cdot \ldots \\
\cdot \cdot \mathbb{I}\left(\left(Y_{n-1}, \tau_{n-1}, \xi_{n-1}\right) \notin B\right) \mathbb{I}\left(\left(Y_{n}, \tau_{n}, \xi_{n}\right) \in B\right) \\
=\sum_{n=0}^{\infty} X_{n}^{*} \mathbb{I}\left(\left(Y_{0}, \tau_{0}\right) \notin C\right) \cdot \ldots \cdot \mathbb{I}\left(\left(Y_{n-1}, \tau_{n-1}\right) \notin C\right) \cdot \mathbb{I}\left(\left(Y_{n}, \tau_{n}\right) \in C\right) \\
=\sum_{n=0}^{\infty} X_{n}^{*} \mathbb{I}(\tilde{\sigma}=n)
\end{aligned}
$$

where

$$
\tilde{\sigma}=\inf \left\{n \geq 0:\left(Y_{n}, \tau_{n}\right) \in C\right\} .
$$

Using the assumptions of the theorem we have

$$
\begin{aligned}
\mathbb{I}\left(\sigma^{*}=\infty\right) \limsup _{n \rightarrow \infty} X_{n}^{*} & =\mathbb{I}\left(\sigma^{*}=\infty\right) \lim _{n \rightarrow \infty}\left(X_{n}^{*} \mathbb{I}\left(\xi_{n}=1\right)\right) \\
& =\prod_{k=0}^{\infty} \mathbb{I}\left(\sigma^{*}>k\right) \lim _{n \rightarrow \infty}\left(X_{n}^{*} \prod_{k=0}^{n} \mathbb{I}\left(\xi_{k}=1\right)\right) \\
& =\prod_{k=0}^{\infty} \mathbb{I}\left(\left(Y_{k}, \tau_{k}, \xi_{k}\right) \notin B\right) \prod_{k=0}^{\infty} \mathbb{I}\left(\xi_{k}=1\right) \lim _{n \rightarrow \infty} X_{n}^{*} \\
& =\lim _{n \rightarrow \infty} X_{n}^{*} \prod_{k=0}^{\infty} \mathbb{I}\left(\left(Y_{k}, \tau_{k}, \xi_{k}\right) \in S\right) \mathbb{I}\left(\left(Y_{k}, \tau_{k}, \xi_{k}\right) \notin B\right) \\
& =\lim _{n \rightarrow \infty} X_{n}^{*} \prod_{k=0}^{\infty} \mathbb{I}\left(\left(Y_{k}, \tau_{k}\right) \notin C\right)=X_{\infty}^{*} \mathbb{I}(\tilde{\sigma}=\infty) .
\end{aligned}
$$

Hence, $X_{\sigma^{*}}^{*}=X_{\tilde{\sigma}}^{*}$. Note that $\tilde{\sigma} \in \mathcal{T}$. Hence, for the optimal Markov time $\sigma^{*}$ there exists the Markov time $\tilde{\sigma} \in \mathcal{T}$ such that $X_{\sigma^{*}}^{*}=X_{\tilde{\sigma}}^{*}$.

Now, note that

$$
E\left(\sup \left|X_{n}^{*}\right|\right) \leq E\left(\sup \left(Y_{n} r\left(\tau_{n}\right)\right)\right)+2 b \leq E\left(\sum_{n=1}^{\infty} Y_{n} r\left(\tau_{n}\right)\right)+2 b<\infty .
$$

Therefore, using (2.1) we see that the assumptions of Theorem 2.2 are satisfied. Hence, the optimal relevant stopping problem with the random horizon 
is equivalent to the optimal stopping problem with the reward sequence

$$
X_{n}=Y_{n} \tilde{r}\left(\tau_{n}\right)+\tilde{\varphi}\left(\tau_{n}\right), \quad n=0,1, \ldots,
$$

where $\tilde{r}(s)=r(s) \bar{F}_{T}(s)$ and $X_{\infty}=\limsup _{n \rightarrow \infty} X_{n}$, in the sense that they give the same optimal mean rewards. Moreover, $\tilde{r}(\cdot)$ satisfies the same conditions as $r(\cdot)$.

To solve the above problem, we will find $\sup _{t \in \mathcal{T}} E\left(X_{t}(u)\right), u \geq 0$, where

$$
X_{n}(u)=Y_{n} \tilde{r}\left(u+\tau_{n}\right)+\tilde{\varphi}\left(u+\tau_{n}\right) .
$$

Note that $E\left(\sup \left(X_{n}(u)\right)^{+}\right)<\infty$, and $\left\{Z_{n, u}\right\}$, where $Z_{n, u}=\left(Y_{n}, u+\tau_{n}\right)$, $n=0,1, \ldots$, is a homogeneous Markov chain with respect to $\left\{\mathcal{F}_{n}\right\}_{n=0}^{\infty}$ and $X_{n}(u)$ is the function of $Z_{n, u}$ only. Hence, using the fact that $Y_{i}$ are i.i.d.

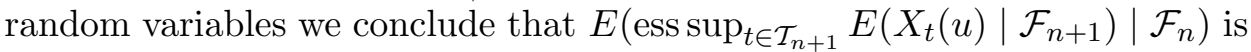
a function of $u+\tau_{n}$ only, say $V\left(u+\tau_{n}\right)$ (see Chow et al. [7, p. 104, Theorem 5.2]). Moreover, from Chow et al. [7, p. 66, Theorem 4.1] we have

$$
\begin{aligned}
\sup _{t \in \mathcal{T}} E\left(X_{t}(u)\right) & =E\left(\max \left\{X_{0}(u), V\left(u+\tau_{0}\right)\right\}\right) \\
& =\max \{d \tilde{r}(u)+\tilde{\varphi}(u), V(u)\} .
\end{aligned}
$$

Therefore, we need to find $V(u)$. Denote $\mathcal{T}_{1}=\{t \in \mathcal{T}: t \geq 1\}$. Note that the Markov time

$$
\sigma_{1}(u)=\inf \left\{n \geq 1: X_{n}(u) \geq V\left(u+\tau_{n}\right)\right\}
$$

is optimal in $\mathcal{T}_{1}$ for the sequence $X_{n}(u), u \geq 0$, i.e. $\sup _{t \in \mathcal{T}_{1}} E\left(X_{t}(u)\right)=$ $E\left(X_{\sigma_{1}(u)}(u)\right)$. From Chow et al. [7, p. 66, Theorem 4.1] we have

$$
\sup _{t \in \mathcal{T}_{1}} E\left(X_{t}(u)\right)=E\left(\operatorname{ess~sup}_{t \in \mathcal{T}_{1}} E\left(X_{t}(u) \mid \mathcal{F}_{1}\right)\right)=V\left(u+\tau_{0}\right)=V(u) .
$$

Proposition 2.3. The function $V(u)$ is continuous on $[0, \infty)$.

Proof. Note that $V(u)<\infty$. First, we will show that for $u_{2}>0$ and $u_{1} \geq 0$ we have

$$
\sup _{t \in \mathcal{T}_{1}}\left|E\left(X_{t}\left(u_{1}\right)\right)-E\left(X_{t}\left(u_{2}\right)\right)\right| \geq\left|\sup _{t \in \mathcal{T}_{1}} E\left(X_{t}\left(u_{1}\right)\right)-\sup _{t \in \mathcal{T}_{1}} E\left(X_{t}\left(u_{2}\right)\right)\right| .
$$

Without loss of generality, we can assume that

$$
\sup _{t \in \mathcal{T}_{1}} E\left(X_{t}\left(u_{1}\right)\right) \geq \sup _{t \in \mathcal{T}_{1}} E\left(X_{t}\left(u_{2}\right)\right) .
$$

Therefore,

$$
\begin{aligned}
& \sup _{t \in \mathcal{T}_{1}}\left|E\left(X_{t}\left(u_{1}\right)\right)-E\left(X_{t}\left(u_{2}\right)\right)\right| \geq\left|E\left(X_{\sigma_{1}\left(u_{1}\right)}\left(u_{1}\right)\right)-E\left(X_{\sigma_{1}\left(u_{1}\right)}\left(u_{2}\right)\right)\right| \\
& \quad=\left|E\left(X_{\sigma_{1}\left(u_{1}\right)}\left(u_{1}\right)\right)-\sup _{t \in \mathcal{T}_{1}} E\left(X_{t}\left(u_{2}\right)\right)+\sup _{t \in \mathcal{T}_{1}} E\left(X_{t}\left(u_{2}\right)\right)-E\left(X_{\sigma_{1}\left(u_{1}\right)}\left(u_{2}\right)\right)\right| \\
& \quad \geq\left|E\left(X_{\sigma_{1}\left(u_{1}\right)}\left(u_{1}\right)\right)-\sup _{t \in \mathcal{T}_{1}} E\left(X_{t}\left(u_{2}\right)\right)\right|=\left|\sup _{t \in \mathcal{T}_{1}} E\left(X_{t}\left(u_{1}\right)\right)-\sup _{t \in \mathcal{T}_{1}} E\left(X_{t}\left(u_{2}\right)\right)\right|,
\end{aligned}
$$


where the last inequality follows from 2.6 and the definition of the supremum. Hence, we have 2.5.

From the assumptions of the model we find that the function $\tilde{\varphi}(\cdot)$ has a finite limit at $+\infty$. Hence, if $\tilde{\varphi}(\cdot)$ is continuous on $[0, \infty)$, it is uniformly continuous. Therefore, for all $\epsilon>0$, there exists $\delta>0$ such that $\mid \tilde{\varphi}\left(u_{1}\right)-$ $\tilde{\varphi}\left(u_{2}\right) \mid<\epsilon$ for all $u_{1}, u_{2}$ with $\left|u_{1}-u_{2}\right|<\delta$. Hence,

$$
\sup _{t \in \mathcal{T}_{1}} E\left|\tilde{\varphi}\left(u_{1}+\tau_{t}\right)-\tilde{\varphi}\left(u_{2}+\tau_{t}\right)\right|<\epsilon .
$$

If $\tilde{\varphi}(\cdot)$ is right-continuous, then

$\sup _{t \in \mathcal{T}_{1}} E\left|\tilde{\varphi}\left(u_{1}+\tau_{t}\right)-\tilde{\varphi}\left(u_{2}+\tau_{t}\right)\right| \leq \sum_{n=1}^{\infty} E\left|\tilde{\varphi}\left(u_{1}+\tau_{n}\right)-\tilde{\varphi}\left(u_{2}+\tau_{n}\right)\right| \leq 2 b\left|u_{1}-u_{2}\right|$.

Now, we will prove that $V(\cdot)$ is continuous on $[0, \infty)$. Let $\epsilon>0$ and fix $u_{1} \in[0, \infty)$. Note that using (2.4) and 2.5 we have

$$
\begin{aligned}
& \left|V\left(u_{1}\right)-V\left(u_{2}\right)\right| \leq \sup _{t \in \mathcal{T}_{1}}\left|E\left(X_{t}\left(u_{1}\right)\right)-E\left(X_{t}\left(u_{2}\right)\right)\right| \\
& \quad \leq \sup _{t \in \mathcal{T}_{1}}\left|E\left(Y_{t}\left(\tilde{r}\left(u_{1}+\tau_{t}\right)-\tilde{r}\left(u_{2}+\tau_{t}\right)\right)\right)\right|+\sup _{t \in \mathcal{T}_{1}}\left|E\left(\tilde{\varphi}\left(u_{1}+\tau_{t}\right)-\tilde{\varphi}\left(u_{2}+\tau_{t}\right)\right)\right| .
\end{aligned}
$$

The first summand above can be estimated as follows:

$$
\begin{aligned}
& \sup _{t \in \mathcal{T}_{1}}\left|E\left(Y_{t}\left(\tilde{r}\left(u_{1}+\tau_{t}\right)-\tilde{r}\left(u_{2}+\tau_{t}\right)\right)\right)\right| \leq \sup _{t \in \mathcal{T}_{1}} E\left(Y_{t}\left|\tilde{r}\left(u_{1}+\tau_{t}\right)-\tilde{r}\left(u_{2}+\tau_{t}\right)\right|\right) \\
& \quad \leq E\left(\sum_{n=1}^{\infty} Y_{n}\left|\tilde{r}\left(u_{1}+\tau_{n}\right)-\tilde{r}\left(u_{2}+\tau_{n}\right)\right|\right)=\mu \int_{0}^{\infty}\left|\tilde{r}\left(u_{1}+x\right)-\tilde{r}\left(u_{2}+x\right)\right| d x \\
& \quad \leq \mu\left|u_{1}-u_{2}\right| .
\end{aligned}
$$

Therefore, if $\tilde{\varphi}(\cdot)$ is continuous on $[0, \infty)$, then

$$
\left|V\left(u_{1}\right)-V\left(u_{2}\right)\right| \leq \mu\left|u_{1}-u_{2}\right|+\epsilon
$$

Hence, taking $0<\delta<\epsilon$, we get $\left|V\left(u_{1}\right)-V\left(u_{2}\right)\right| \leq(\mu+1) \epsilon$, and so $V(\cdot)$ is continuous at $u_{1}$. If $\tilde{\varphi}(\cdot)$ is only right-continuous, then

$$
\left|V\left(u_{1}\right)-V\left(u_{2}\right)\right| \leq \mu\left|u_{1}-u_{2}\right|+2 b\left|u_{1}-u_{2}\right| \text {. }
$$

Hence, taking $0<\delta<\epsilon$, we get $\left|V\left(u_{1}\right)-V\left(u_{2}\right)\right| \leq(\mu+2 b) \epsilon$ and again $V(\cdot)$ is continuous at $u_{1}$.

From 2.3 and Proposition 2.3, we have

Corollary 2.4 .

$$
\sup _{t \in \mathcal{T}} E\left(X_{t}\right)=\max \left\{d \tilde{r}(0)+\tilde{\varphi}(0), \lim _{u \rightarrow 0^{+}} V(u)\right\} .
$$

Denote

$$
y(s)=\frac{V(s)-\tilde{\varphi}(s)}{\tilde{r}(s)} \quad \text { for } s \in[0, \tilde{U})
$$


and $y(s)=0$ for $s \geq \tilde{U}$. Set $\bar{F}(x)=1-F(x), H(x)=\int_{x}^{\infty} s d F(s)$ and $f_{V, u}(v)=P\left(\tau_{\sigma_{1}(u)}>v\right)$. Note that in the notation $f_{V, u}(v)$ we use $V$ to emphasize that the distribution of $\tau_{\sigma_{1}(u)}$ depends on the function $V(\cdot)$. The ensumptions of the model ensure that there exist $k<\infty$ and $\left\{s_{1}, \ldots, s_{k}\right\}$ such that $0<s_{1}<\cdots<s_{k}<s_{k+1}, s_{0}=0, s_{k+1}=\tilde{U}$, the function $y(\cdot)$ is continuous in each of the intervals $\left(v_{i}, v_{i+1}\right), i=0,1, \ldots, k$, and for each $z \in\left(v_{i}, v_{i+1}\right), i=0,1, \ldots, k$,

$$
\lim _{h \rightarrow 0} F\left(\inf _{x \in(-h, 0]} y(z+x)\right)=\lim _{h \rightarrow 0} F\left(\inf _{x \in(0, h]} y(z+x)\right)=F(y(z)) .
$$

Moreover, $f_{V, u}(\cdot)$ is continuous on $[0, \infty)$ and $f_{V, u}(0)=1$. Therefore, considerations similar to those in Chow et al. [7, pp. 114-115] for $u \in[0, \tilde{U})$ give

$$
f_{V, u}(v)=\left\{\begin{array}{lc}
\exp \left(-\int_{u}^{u+v} \bar{F}\left(y\left(v^{\prime}\right)\right) d v^{\prime}\right), & 0<v \leq \tilde{U}-u, \\
f_{V, u}(\tilde{U}-u) \exp \left(-\int_{\tilde{U}}^{u+v} \mathbb{I}\left(\tilde{\varphi}\left(v^{\prime}\right) \geq V\left(v^{\prime}\right)\right) d v^{\prime}\right), & v>\tilde{U}-u .
\end{array}\right.
$$

Moreover, for $u \geq \tilde{U}$ and $v \geq 0$ we have

$$
f_{V, u}(v)=\exp \left(-\int_{u}^{u+v} \mathbb{I}\left(\tilde{\varphi}\left(v^{\prime}\right) \geq V\left(v^{\prime}\right)\right) d v^{\prime}\right) .
$$

Note that

$$
\begin{aligned}
V(u) & =E\left(Y_{\sigma_{1}(u)} \tilde{r}\left(u+\tau_{\sigma_{1}(u)}\right)+\tilde{\varphi}\left(u+\tau_{\sigma_{1}(u)}\right)\right) \\
& =E\left(\tilde{\varphi}\left(u+\tau_{\sigma_{1}(u)}\right)+\tilde{r}\left(u+\tau_{\sigma_{1}(u)}\right) E\left(Y_{\sigma_{1}(u)} \mid \sigma_{1}(u)\right)\right) .
\end{aligned}
$$

Moreover, for $u \in[0, \tilde{U})$ and $\tau_{\sigma_{1}(u)} \in[0, \tilde{U}-u)$, the conditional distribution of $Y_{\sigma_{1}(u)}$ given $\sigma_{1}(u)$ is the same as the conditional distribution of $Y$ given $\left\{Y \geq y\left(u+\tau_{\sigma_{1}(u)}\right)\right\}$, where $Y$ and $\tau_{\sigma_{1}(u)}$ are independent and $Y$ has the distribution function $F$. Hence, for $u \in\left(s_{i}, s_{i+1}\right), i=0, \ldots, k$, we have

$$
\begin{aligned}
V(u)= & \int_{0}^{\tilde{U}-u}\left(\tilde{\varphi}(u+v)+\frac{\tilde{r}(u+v) \int_{y(u+v)}^{\infty} y d F(y)}{\bar{F}(y(u+v))}\right) \cdot \frac{\partial}{\partial v}\left(1-f_{V, u}(v)\right) d v \\
& +\int_{\tilde{U}-u}^{\infty} \tilde{\varphi}(u+v) \cdot \frac{\partial}{\partial v}\left(1-f_{V, u}(v)\right) d v .
\end{aligned}
$$

Using (2.8) we obtain, for $u \in\left(s_{i}, s_{i+1}\right), i=0, \ldots, k$, 


$$
\begin{aligned}
V(u)= & \int_{u}^{\tilde{U}}(\tilde{\varphi}(v) \bar{F}(y(v))+\tilde{r}(v) H(y(v))) f_{V, u}(v-u) d v \\
& +\int_{\tilde{U}}^{\infty} \tilde{\varphi}(v) f_{V, u}(v-u) \mathbb{I}(\tilde{\varphi}(v) \geq V(v)) d v .
\end{aligned}
$$

Moreover, for $u \geq \tilde{U}$ we have

$$
\begin{aligned}
& V(u)=E\left(\tilde{\varphi}\left(u+\tau_{\sigma_{1}(u)}\right)\right) \\
& =\int_{u}^{\infty} \tilde{\varphi}(v) \mathbb{I}(\tilde{\varphi}(v) \geq V(v)) \exp \left(-\int_{u}^{v} \mathbb{I}\left(\tilde{\varphi}\left(v^{\prime}\right) \geq V\left(v^{\prime}\right)\right) d v^{\prime}\right) d v .
\end{aligned}
$$

REMARK 2.5. Note that for $x \geq \tilde{U}$ we have

$$
\mathbb{I}(\tilde{\varphi}(x) \geq V(x))= \begin{cases}1 & \text { if } \tilde{\varphi}(\cdot) \text { is non-increasing on }(\tilde{U}, \infty), \\ 0 & \text { if } \tilde{\varphi}(\cdot) \text { is increasing on }(\tilde{U}, \infty) .\end{cases}
$$

Therefore, the right-hand site of 2.11 is independent of $V(\cdot)$ and consequently $V(u)$ for $u \geq \tilde{U}$ is uniquely determined by 2.11. Hence,

$$
V(\tilde{U})= \begin{cases}\int_{\tilde{U}}^{\infty} \tilde{\varphi}(v) \exp (\tilde{U}-v) d v \\ 0 & \text { if } \tilde{\varphi}(\cdot) \text { is non-increasing on }(\tilde{U}, \infty), \\ & \text { if } \tilde{\varphi}(\cdot) \text { is increasing on }(\tilde{U}, \infty) .\end{cases}
$$

The proof of the theorem below is based on Chow et al. [7, pp. 115-116] and on the proof of Theorem 2.1(c) from Stadje [25].

TheOREM 2.6.

(i) Let $\tilde{V}(\cdot)$ be a continuous function on $[0, \infty)$ such that $\tilde{V}(u)=V(u)$ for $u \geq \tilde{U}$. Then $\tilde{V}(\cdot)$ satisfies 2.10 for $u \in\left(s_{i}, s_{i+1}\right), i=0, \ldots, k$, if and only if

$$
\frac{d}{d u} \tilde{V}(u)=\bar{F}(\tilde{y}(u)) \tilde{V}(u)-\tilde{r}(u) H(\tilde{y}(u))-\tilde{\varphi}(u) \bar{F}(\tilde{y}(u))
$$

for $u \in\left(s_{i}, s_{i+1}\right), i=0, \ldots, k$, where $\tilde{y}(s)=(\tilde{V}(s)-\tilde{\varphi}(s)) / \tilde{r}(s)$ for $s \in[0, \tilde{U})$.

(ii) There exists exactly one continuous function $\tilde{V}(\cdot)$ satisfying 2.14 for $u \in\left(s_{i}, s_{i+1}\right), i=0, \ldots, k$, such that $\tilde{V}(\tilde{U})=V(\tilde{U})$.

Proof. (i) Differentiating both sides of (2.10) with respect to $u$ in each of the intervals $\left(s_{i}, s_{i+1}\right), i=0, \ldots, k$, we see that if $\tilde{V}(\cdot)$ satisfies 2.10, then $\tilde{V}(\cdot)$ has to satisfy 2.14 . Now, suppose that the continuous function $\tilde{V}(\cdot)$ satisfies 2.14 on $\left(s_{i}, s_{i+1}\right), i=0, \ldots, k$, and $\tilde{V}(\tilde{U})=V(\tilde{U})$. For all 
$u \in[0, \tilde{U}]$, define $V_{1}(u)$ by

$$
\begin{aligned}
V_{1}(u)= & \int_{u}^{\tilde{U}}(\tilde{\varphi}(v) \bar{F}(\tilde{y}(v))+\tilde{r}(v) H(\tilde{y}(v))) f_{\tilde{V}, u}(v-u) d v \\
& +\int_{\tilde{U}}^{\infty} \tilde{\varphi}(v) f_{\tilde{V}, u}(v-u) \mathbb{I}(\tilde{\varphi}(v) \geq \tilde{V}(v)) d v .
\end{aligned}
$$

Note that $V_{1}(u)$ is continuous for $u \in[0, \tilde{U}]$. We want to show that $V_{1}(u)=$ $\tilde{V}(u)$ for $u \in[0, \tilde{U}]$. Note that $\left|(\tilde{\varphi}(v) \bar{F}(\tilde{y}(v))+\tilde{r}(v) H(\tilde{y}(v))) f_{\tilde{V}, u}(v-u)\right| \leq$ $2 b+\mu$. Hence, taking $u \rightarrow \tilde{U}$ in 2.15 and using the assumption that $\tilde{V}(u)=$ $V(u)$ for $u \geq \tilde{U}$ and 2.11) we see that $V_{1}(\tilde{U})=V(\tilde{U})=\tilde{V}(\tilde{U})$. Moreover, differentiating (2.15) in each of the intervals $\left(s_{i}, s_{i+1}\right), i=0, \ldots, k$, we obtain

$$
\frac{d}{d u} V_{1}(u)=\bar{F}(\tilde{y}(u)) V_{1}(u)-\tilde{r}(u) H(\tilde{y}(u))-\tilde{\varphi}(u) \bar{F}(\tilde{y}(u)) .
$$

Assume that $V_{1}\left(u_{0}\right) \neq \tilde{V}\left(u_{0}\right)$ at some point $u_{0} \in\left(s_{k}, \tilde{U}\right)$. Let $u_{1}$ be the first point after $u_{0}$ such that $u_{1} \in\left(s_{k}, \tilde{U}\right\rceil$ and $V_{1}\left(u_{1}\right)=\tilde{V}\left(u_{1}\right)$. From 2.16$)$ and the assumption that $\tilde{V}(\cdot)$ satisfies 2.14 we obtain $d\left(V_{1}(u)-\tilde{V}(u)\right) / d u=$ $\bar{F}(\tilde{y}(u))\left(V_{1}(u)-\tilde{V}(u)\right)$. Hence, for $u \in\left(u_{0}, u_{1}\right)$ we have

$$
\ln \left|V_{1}(u)-\tilde{V}(u)\right|=\ln \left|V_{1}\left(u_{0}\right)-\tilde{V}\left(u_{0}\right)\right|+\int_{u_{0}}^{u} \bar{F}\left(\tilde{y}\left(u^{\prime}\right)\right) d u^{\prime} .
$$

For $u \rightarrow u_{1}$ the left-hand side of the above equation goes to $-\infty$ while the right-hand side is finite. This contradiction gives that $V_{1}(u)=\tilde{V}(u)$ for $u \in\left(s_{k}, \tilde{U}\right)$. From the continuity of the functions $V_{1}(u)$ and $\tilde{V}(u)$ we obtain $V_{1}(u)=\tilde{V}(u)$ for $u \in\left[s_{k}, \tilde{U}\right]$. Now, by recursion we conclude that $V_{1}(u)=\tilde{V}(u)$ in each of the intervals $\left[s_{i}, s_{i+1}\right], i=k, \ldots, 0$.

(ii) Let

$$
g(x)=\bar{F}(x)(x \tilde{r}(u)+\tilde{\varphi}(u))-\tilde{r}(u) H(x)-\tilde{\varphi}(u) \bar{F}(x)
$$

and

$$
G(u, \tilde{V})=g((\tilde{V}-\tilde{\varphi}(u)) / \tilde{r}(u)) .
$$

Note that $G(u, \tilde{V}(u))$ is equal to the right-hand side of 2.14). Moreover, in each of the intervals of differentiability of $F$, we have $d g(x) / d x=\bar{F}(x) \tilde{r}(u)$ and consequently $d G(u, \tilde{V}) / d \tilde{V}=\bar{F}((\tilde{V}-\tilde{\varphi}(u)) / \tilde{r}(u)) \leq 1$. Therefore,

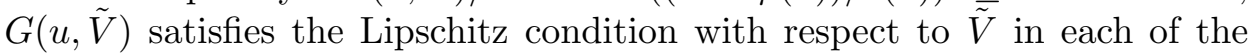
intervals of differentiability of $F$. Now, note that $|\tilde{\varphi}(\cdot)| \leq 2 b$, hence from (2.10) we see that

$$
|V(u)| \leq \int_{0}^{\tilde{U}}(2 b+\mu) d v+\int_{\tilde{U}}^{\infty} 2 b \exp (\tilde{U}-v) d v=(2 b+\mu) \tilde{U}+2 b \quad \text { for } u \in[0, \tilde{U}] .
$$


Moreover,

$$
|G(u, \tilde{V})| \leq|\tilde{V}|+\mu+2 b \leq(2 b+\mu) \tilde{U}+4 b+\mu .
$$

Hence, the assumptions of the Picard-Lindelöf theorem (see Lindelöf [19] or Coddington and Levinson [8, Theorem 1.3.1]) are satisfied in each of the intervals in which $\tilde{r}$ is continuous and $F$ is differentiable. Therefore, using the fact that $\tilde{V}(u)$ is continuous, we conclude that 2.14 has exactly one solution on $[0, \tilde{U}]$.

REMARK. The optimal Markov time in $\mathcal{T}$ for the sequence $X_{n}$ is

$$
\sigma(0)=\inf \left\{n \geq 0: X_{n} \geq V\left(\tau_{n}\right)\right\} .
$$

REMARK. Since the distribution function of $\tau_{\sigma_{1}(0)}$ is given in 2.8 and 2.9 , we can compute the mean waiting time for the optimal Markov time, that is,

$$
\begin{aligned}
E\left(\tau_{\sigma(0)}\right) & =E\left(\tau_{0} \mathbb{I}\left(X_{0} \geq V(0)\right)\right)+E\left(\tau_{\sigma_{1}(0)} \mathbb{I}\left(X_{0}<V(0)\right)\right) \\
& =\mathbb{I}(d \tilde{r}(0)+\tilde{\varphi}(0)<V(0)) E\left(\tau_{\sigma_{1}(0)}\right) .
\end{aligned}
$$

Remark. Assuming that $P(T=U)=1$, we obtain the solution of the optimal stopping problem without random horizon.

\section{Generalizations and examples}

3.1. Optimal stopping problem with infinite horizon. Suppose that all assumptions of Section 2 are satisfied with one exception: assume that $\tilde{U}=\infty$. Moreover, assume that

$$
\int_{0}^{\infty} r(s) d s<\infty \quad \text { and } \quad \int_{0}^{\infty}|\tilde{\varphi}(s)| d s<\infty .
$$

Hence, for $u \geq 0$ we have $E\left(\sup \left|X_{n}^{*}(u)\right|\right)<\infty$. Note that

$$
\sup _{t^{*} \in \mathcal{T}^{*}} E\left(\tilde{X}_{t^{*}}^{*}\right)=\sup _{t \in \mathcal{T}} E\left(X_{t}\right)=\max \{d \tilde{r}(0)+\tilde{\varphi}(0), V(0)\},
$$

where $V(\cdot)$ is continuous on $[0, \infty)$. In this case, equation 2.10 has the following form: for $u \in\left(s_{i}, s_{i+1}\right), i=0, \ldots, k$,

$$
V(u)=\int_{u}^{\tilde{U}}(\tilde{\varphi}(v) \bar{F}(y(v))+\tilde{r}(v) H(y(v))) f_{V, u}(v-u) d v,
$$

where $y(s)=(V(s)-\tilde{\varphi}(s)) / \tilde{r}(s)$ for $s \geq 0$, and

$$
f_{V, u}(v)=\exp \left(-\int_{u}^{u+v} \bar{F}\left(y\left(v^{\prime}\right)\right) d v^{\prime}\right) \quad \text { for } v \geq 0
$$


THEOREM 3.1.

(i) A continuous function $\tilde{V}(\cdot)$ satisfies 3.2 for $u \in\left(s_{i}, s_{i+1}\right), i=$ $0, \ldots, k$, if and only if

$$
\frac{d}{d u} \tilde{V}(u)=\bar{F}(\tilde{y}(u)) \tilde{V}(u)-\tilde{r}(u) H(\tilde{y}(u))-\tilde{\varphi}(u) \bar{F}(\tilde{y}(u))
$$

for $u \in\left(s_{i}, s_{i+1}\right), i=0, \ldots, k$, and

$$
\lim _{u \rightarrow \infty} \tilde{V}(u)=0 .
$$

(ii) If a continuous function $\tilde{V}(u)$ satisfies 3.4 for $u \in\left(s_{i}, s_{i+1}\right), i=$ $0, \ldots, k$, and 3.5, then $V\left(\tau_{n}\right)=\tilde{V}\left(\tau_{n}\right), n=0,1, \ldots$

Proof. (i) The proof is analogous to the proof of Theorem 2.6 (see also Chow et al. [7, pp. 116-118]). Note that in this case we use the assumption (3.1).

(ii) The proof is analogous to that in Chow et al. [7, pp. 116-118] (see also Krasnosielska [17] for more details). Note only that in the present problem, the random variable $W(u)$ considered in Krasnosielska [17] has the form $W(u)=2 b+\sum_{n=0}^{\infty}\left|Y_{n} r(u)\right|$.

3.2. Non-homogeneous Poisson process. Since a non-homogeneous Poisson process with intensity function $p(\cdot)$ can be reduced to a homogeneous Poisson process (see Chow et al. [7, pp. 113-114])), we can consider the problem with a homogeneous Poisson process without loss of generality.

3.3. Examples. Let $U<\infty, P(T=U)=1, r(u)=1$ if $0 \leq u<U$, and $r(u)=0$ otherwise. Moreover, let $\left\{Y_{n}\right\}_{n=1}^{\infty}$ be a sequence of i.i.d. random variables with exponential distribution with mean 1 and $Y_{0}=0$.

(a) If $\tilde{\varphi}(u)=0$ for all $u \geq 0$, then $\sup _{t \in \mathcal{T}} E\left(X_{t}\right)=V(0)=\ln (1+U)$.

(b) If $\tilde{\varphi}(u)=a-c u$ for all $u \in[0, U)$ and $\tilde{\varphi}(u)=a-c U$ for all $u \geq U$, where $c \in \mathbb{R}$ and $a \in \mathbb{R}$, then from (2.13) we have $V(U)=a-c U$ and from the continuity of $V(\cdot)$ we have $y(U)=0$. Note that equation 2.14 is equivalent to $d y(u) / d u=c-\exp (-y(u))$. Hence,

$$
y(u)=\ln \left(\frac{1+c \exp (-c U+c u)-\exp (-c U+c u)}{c}\right) .
$$

Since $\tilde{\varphi}(0)=a$ and $r(0)=1$, we have $\sup _{t \in \mathcal{T}} E\left(X_{t}\right)=\max \{a, y(0)+a\}$.

\section{Conclusion}

4.1. Related papers. The Elfving problem has been modified in various ways. Stadje [25] generalized the Elfving problem to a multi-stopping problem. Albright [3] considered an $n$-person assignment problem. Ferenstein and Krasnosielska [15] considered a multi-person game with priorities 
and a reward structure as in the Elfving problem. David and Yechiali [1] considered a problem with a renewal process. Parlar et al. 20. generalized the Elfving problem to a Markov chain model. A model with random starting time based on the Elfving problem was analyzed by Krasnosielska [17. A formula for the mean waiting time for the optimal Markov time in the Elfving problem was given by Krasnosielska [18.

Samuel-Cahn 24 considered an optimal stopping problem with an independent random horizon $M$ and a known number of offers. The problem was translated to a problem with discounting. Ferenstein and Krasnosielska [16] generalized the theorem of [24] concerning a stopping problem with a random horizon to the Poisson case and applied it to the Elfving problem.

Cowan and Zabczyk [9] considered a continuous-time version of the secretary problem in which the offers appear at jump times of a Poisson process up to a fixed time $T$. The secretary problem with a renewal process and random horizon was analyzed in Bojdecki [5]. Optimal stopping problems with costs based on relative ranks were considered by Bartoszyński and Govindarajulu [4] and Szajowski [26].

Various applications of optimal stopping problems with a Poisson process were considered by many authors (see Sakaguchi [22], 23] and Ferenstein and Krasnosielska [16]). An interesting application to kidney allocation for transplant was presented by Righter [21. She considered a problem with a random deadline. In her model activities (patients) and resources (kidneys) have different values, hence she assumed that the probability that a transplant is successful is the product of the kidney value and the patient value. Alagoz et al. [1, [2] also considered a medical application of an optimal stopping problem. They were interested in finding an optimal policy for patients with end-stage liver disease. They formulated a Markov decision process model in which the state of the process is described by the patient's condition and the organ's quality. The model with a penalty function depending on the patient's health and involving a living donor was introduced by Alagoz et al. [1. This function is in units of the total expected discounted reward.

Optimal stopping problems for more than one patient were considered by David [10] and David and Yechiali [12] and [13].

4.2. Other applications. The random variables $Y$ can also be interpreted as the quality of the organ for transplant. The quality depends not only on the degree of histocompatibility between the organ and the recipient but possibly also on the age and condition of the donor, cold ischemia time and anatomic anomalies of the organ and the patient. It is also possible to consider other organs for transplant, for example liver.

However, the most popular application of such a model is asset selling problem (for example, selling a car or a house). In this case $Y$ 's can be 
interpreted as the value of the offer, $r(\cdot)$ as a discount function, $\xi$ as a random time when a feeling of risk appears (as in the paper of Szajowski [26]), $c_{1}(\cdot)$ as a cost function (for example related to taxes), $c_{2}(\cdot)$ as a cost of stress, $T$ as a time after which it is impossible to obtain any offer (for example a time of collapsing of the house). Note that $\tilde{X}_{n}^{*}$ is a reward for the decision maker who accepts offer $Y_{n}$ at time $\tau_{n}$.

4.3. Further research. In this paper we presented the problem from the patient's (or physician's) point of view and we introduced a function related to the level of risk aversion of the patient assuming that the level of acceptance of risk can vary in time. This assumption is justified because at an advanced stage of kidney disease, the only possible treatment is transplantation and the stage is to large extent dependent on how long the patient is ill. On the other hand, it could be valuable to extend the model by introducing a function related to the information about the stage of the disease the patient is in. Therefore, further research may consider this modification. Moreover, one can take into the account the patient's place on the waiting list.

Acknowledgments. The author wishes to express her special thanks to Professor E. Ferenstein for her helpful advice concerning this work, the referee of the first version of this manuscript for careful reading and valuable suggestions concerning the proof of Theorem 2.2, and the referee of the present version for constructive questions and suggestions related to application of the model in transplantology and help in reorganization of the introduction.

This work has been partially supported by the European Union in the framework of European Social Fund through the Warsaw University of Technology Development Programme and the European Social Fund and National Budget in the framework of Integrated Operational Programme for Regional Development (ZPORR), Action 2.6: 'Regional Innovation Strategies and Transfer of Knowledge' through the Mazovian Voivodeship's 'Mazovian PhD Student Scholarship'.

\section{References}

[1] O. Alagoz, L. M. Maillart, A. J. Schaefer and M. S. Roberts, Choosing among living-donor and cadaveric livers, Management Sci. 53 (2007), 1702-1715.

[2] - - - - - - Determining the acceptance of cadaveric livers using an implicit model of the waiting list, Oper. Res. 55 (2007), 24-36.

[3] S. Ch. Albright, Optimal sequential assignments with random arrival times, Management Sci. 21 (1974), 60-67.

[4] R. Bartoszyński and Z. Govindarajulu, The secretary problem with interview cost, Sankhyā B 40 (1978), 11-28. 
[5] T. Bojdecki, On optimal stopping of independent random variables appearing according to a renewal process, with random time horizon, Bol. Soc. Mat. Mexicana 22 (1977), 35-40.

[6] Ch. A. K. Y. Chong, A. Gulamhussein, A. J. Heathcote, L. Lilly, M. Sherman, G. Naglie and M. Krahn, Health-state utilities and quality of life in hepatitis $C$ paients, Amer. J. Gastroenterology 98 (2003), 630-638.

[7] Y. S. Chow, H. Robbins and D. Siegmund, Great Expectations: The Theory of Optimal Stopping, Houghton Mifflin, Boston, 1971.

[8] E. A. Coddington and N. Levinson, Theory of Ordinary Differential Equations, McGraw-Hill, New York, 1955.

[9] R. Cowan and J. Zabczyk, An optimal selection problem associated with the Poisson process, Theory Probab. Appl. 23 (1978), 584-592.

[10] I. David, A sequential assignment match process with general renewal arrival times, Probab. Engrg. Inform. Sci. 9 (1995), 475-492.

[11] I. David and U. Yechiali, A time-dependent stopping problem with application to live organ transplants, Oper. Res. 33 (1985), 491-504.

[12] - - -, One-attribute sequential assignment match processes in discrete time, ibid. 43 (1995), 879-884.

[13] -, -, Sequential assignment match processes with arrivals of candidates and offers, Probab. Engrg. Inform. Sci. 4 (1990), 413-430.

[14] G. Elfving, A persistency problem connected with a point process, J. Appl. Probab. 4 (1971), 77-89.

[15] E. Z. Ferenstein and A. Krasnosielska, Nash equilibrium in a game version of the Elfving problem, in: Advances in Dynamic Games and Their Applications, P. Bernhard et al. (eds.), Birkhäuser, 2009, 399-414.

[16] - - - A version of the Elfving optimal stopping time problem with random horizon, in: Game Theory and Applications 14, Nova Science Publ., to appear.

[17] A. Krasnosielska, A version of the Elfving problem with random starting time, Statist. Probab. Letters 79 (2009), 2429-2436.

[18] -, On the Elfving problem, Decyzje 9 (2008), 101-114 (in Polish).

[19] E. Lindelöf, Sur l'application de la méthode des approximations successives aux équations différentielles ordinaires du premier ordre, C. R. Acad. Sci. Paris 114 (1894), 454-457.

[20] M. Parlar, D. Perry and W. Stadje, Optimal shopping when the sales are onA Markovian full-information best-choice problem, Stoch. Models 23 (2007), 351371.

[21] R. Righter, A resource allocation problem in a random environment, Oper. Res. 37 (1989), 329-338.

[22] M. Sakaguchi, Optimal stopping problem for randomly arriving offers, Math. Japon. 21 (1976), 201-217.

[23] - A sequential stochastic assignment problem with an unknown number of jobs, ibid. 29 (1984), 141-152.

[24] E. Samuel-Cahn, Optimal stopping with random horizon with application to the full-information best-choice problem with random freeze, J. Amer. Statist. Assoc. 91 (1996), 357-364.

[25] W. Stadje, An optimal k-stopping problem for the Poisson process, in: Mathematical Statistics and Probability Theory, Vol. B (Bad Tatzmannsdorf, 1986), B. P. Bauer et al. (eds.), Reidel, 1987, 231-244.

[26] K. Szajowski, A rank-based selection with cardinal payoffs and a cost of choice, Math. Japon. 69 (2009), 285-293. 
[27] UNOS 2008, Organ Distribution: Allocation of Deceased Kidneys, http://www.unos.org/PoliciesandBylaws2/policies/pdfs/policy_7.pdf.

Anna Krasnosielska

Faculty of Mathematics and Information Science

Warsaw University of Technology

Pl. Politechniki 1

00-661 Warsaw, Poland

E-mail: akrasno@mini.pw.edu.pl

Received on 2.6.2009;

revised version on 23.12.2009 